\title{
A RELATION BETWEEN HIGHER-RANK PT STABLE OBJECTS AND QUOTIENTS OF COHERENT SHEAVES
}

\author{
JASON LO
}

\begin{abstract}
On a smooth projective threefold, we construct an essentially surjective functor $\mathcal{F}$ from a category of two-term complexes to a category of quotients of coherent sheaves, and describe the fibers of this functor. Under a coprime assumption on rank and degree, the domain of $\mathcal{F}$ coincides with the category of higher-rank PT stable objects, which appear on one side of Toda's higher-rank DT/PT correspondence formula. The codomain of $\mathcal{F}$ is the category of objects that appear on one side of another correspondence formula by Gholampour-Kool, between the generating series of topological Euler characteristics of two types of quot schemes.
\end{abstract}

\section{CONTENTS}

1. Introduction

2. Preliminaries

3. The dualising functor

4. A functor taking objects to morphisms

5. Essential surjectivity of the functor $\mathcal{F}$

6. Fibers of the functor $\mathcal{F}$

References

\section{INTRODUCTION}

On a smooth projective threefold $X$, Gholampour-Kool computed the generating series of some moduli spaces of slope stable sheaves of homological dimension at most one [5]. An integral part of their argument was the following counting formula, where $\operatorname{Quot}_{X}(-, n)$ denotes the quot scheme of length- $n$ quotients of a coherent sheaf, $e(-)$ denotes the topological Euler characteristic, and $M(q)=$ $\prod_{n=1}^{\infty} \frac{1}{\left(1-q^{n}\right)^{n}}$ is the MacMahon fuction:

Theorem 1.1. [5, Theorem 1.1] For any rank $r$ torsion-free sheaf $F$ of homological dimension at most 1 on a smooth projective threefold $X$, we have

$$
\sum_{n=0}^{\infty} e\left(\operatorname{Quot}_{X}(F, n)\right) q^{n}=M(q)^{r e(X)} \sum_{n=0}^{\infty} e\left(\operatorname{Quot}_{X}\left(\mathcal{E} x t^{1}\left(F, \mathcal{O}_{X}\right), n\right)\right) q^{n} .
$$

On the other hand, on a smooth projective Calabi-Yau threefold $X$, Toda proved a correspondence formula between higher-rank Donaldson-Thomas (DT) and Pandharipande-Thomas (PT) invariants. While DT invariants virtually count slope stable sheaves on $X$, PT invariants count PT stable objects in the derived category of coherent sheaves $D^{b}(X)=D^{b}(\operatorname{Coh}(X))$ on $X$. PT stability is a type

2010 Mathematics Subject Classification. Primary 14J30; Secondary: 14D23.

Key words and phrases. stable pair, PT stable object, quot scheme. 
of polynomial stability on $D^{b}(X)$ in the sense of Bayer [1]; a rank-one PT stable object of trivial determinant is exactly a stable pair

$$
\mathcal{O}_{X} \stackrel{s}{\rightarrow} F
$$

in the sense of Pandharipande-Thomas [13], which we call a PT stable pair, where $F$ is a pure 1dimensional sheaf and the cokernel of the morphism of sheaves $s$ is 0-dimensional. The properties of PT stable objects were studied and their moduli spaces constructed by the author in [9, 11, 10]. For any ample divisor $\omega$ on $X$ and any $(r, D,-\beta,-n) \in H^{0}(X) \oplus H^{2}(X) \oplus H^{4}(X) \oplus H^{6}(X)$ where $r \geq 1$ with $r, D \omega^{2}$ coprime, let us write $\mathrm{DT}(r, D,-\beta,-n)$ to denote the DT invariant virtually counting $\mu_{\omega}$-stable sheaves of Chern character $(r, D,-\beta,-n)$, and $\mathrm{PT}(r, D,-\beta,-n)$ to denote the PT invariant virtually counting PT stable objects of that Chern character. Then Toda's correspondence formula reads:

Theorem 1.2. [17, Theorem 1.2] For a fixed $(r, D, \beta)$, we have

$$
\sum_{6 n \in \mathbb{Z}} \mathrm{DT}(r, D,-\beta,-n) q^{n}=M\left((-1)^{r} q\right)^{r e(X)} \sum_{6 n \in \mathbb{Z}} \mathrm{PT}(r, D,-\beta,-n) q^{n} .
$$

The case $(r, D)=(1,0)$ of the formula (1.2.1), i.e. rank-one DT/PT correspondence, was first conjectured in [13] and first proved by Bridgeland [2]. Toda also gave a proof under an assumption on the local structure of the moduli stacks involved [16]; the assumption was later removed in [17].

In this article, we describe a relation between the objects that appears on the right-hand side of Gholampour-Kool's formula (1.1.1) and the objects that appears on the right-hand side of Toda's formula (1.2.1). More precisely, on a smooth projective threefold $X$, we define a category $\mathcal{E}_{0}$ of 2-term complexes in $D^{b}(X)$ with cohomology at degrees $-1,0$, that contains all the PT semistable objects in $D^{b}(X)$. The category $\mathcal{E}_{0}$ also contains all the 'frozen triples' in the sense of Sheshmani [15], which gives an alternative approach for generalising Pandharipande-Thomas' stable pairs (1.1.2) to higher ranks. We write $\operatorname{Mor}(\operatorname{Coh}(X))$ to denote the category where the objects are morphisms of coherent sheaves on $X$, and morphisms are given by commutative squares in $\operatorname{Coh}(X)$. For any coherent sheaf $A$ on $X$, let us write $\mathcal{S}(A)$ to denote the full subcategory of $\mathcal{M o r}(\operatorname{Coh}(X))$ consisting of objects of the form $A \stackrel{q}{\rightarrow} Q$ where $Q$ is a 0-dimensional sheaf, and $q$ is a surjection of sheaves. We construct a (contravariant) functor

$$
\mathcal{F}: \mathcal{E}_{0} \rightarrow \coprod_{F \in \operatorname{Coh}(X), \operatorname{hd}(F) \leq 1} \mathcal{S}\left(\mathcal{E} x t^{1}\left(F, \mathcal{O}_{X}\right)\right)^{o p}
$$

(see Definition 5.4) and prove our main result:

Theorem 1.3. (Theorem 5.9) The functor $\mathcal{F}$ is essentially surjective. If we fix an ample class on $X$, fix $r \in H^{0}(X), D \in H^{2}(X)$ such that $r, \omega^{2} D$ are coprime, restrict the domain of $\mathcal{F}$ to $P$ T stable objects $E$ with $\operatorname{ch}_{0}(E)=-r, \operatorname{ch}_{1}(E)=-D$ and restrict the codomain by requiring $F$ above to be $\mu_{\omega}$-stable with $\operatorname{ch}_{0}(F)=r, \operatorname{ch}_{1}(F)=D$, then the restriction of $\mathcal{F}$ is also essentially surjective.

In Section 6 , we analyse the fibers of the functor $\mathcal{F}$. We describe how to enumerate all the objects in a given fiber of $\mathcal{F}$ in Lemma 6.2. For two objects $E, \bar{E}$ of $\mathcal{E}_{0}$, we pin down the difference between $E, \bar{E}$ being isomorphic in $D^{b}(X)$ and $\mathcal{F}(E), \mathcal{F}(\bar{E})$ being isomorphic in $\mathcal{M o r}(\operatorname{Coh}(X))$. Finally, we recall a construction mentioned in Gholampour-Kool's work in which a sheaf quotient $\mathcal{E} x t^{1}\left(I_{C}, \mathcal{O}_{X}\right) \rightarrow Q$, where $I_{C}$ is the ideal sheaf of a Cohen-Macaulay curve $C$ on $X$ and $Q$ is a 0-dimensional sheaf, can be used to construct a PT stable pair (i.e. a rank-one PT stable object). We generalise this construction to higher ranks in 6.8, so that given a higher-rank sheaf quotient, we produce a higher-rank PT stable object. We end the article with Lemma 6.9, which compares this higher-rank construction and the functor $\mathcal{F}$ constructed in Section 5 ,

1.1. Acknowledgements. The author would like to thank Yunfeng Jiang, Martijn Kool and Zhenbo Qin for answering his various questions, and Jun Li and Ziyu Zhang for helpful discussions. 


\section{Preliminaries}

2.1. Notation. Unless otherwise stated, we will write $X$ for a smooth projective threefold in this article, $\operatorname{Coh}(X)$ for the category of coherent sheaves on $X$, and $D^{b}(X)=D^{b}(\operatorname{Coh}(X))$ for the bounded derived category of coherent sheaves on $X$.

2.1. For any category $\mathcal{C}$, we will write $\operatorname{Mor}(\mathcal{C})$ to denote the category of morphisms in $\mathcal{C}$. That is, the objects of $\operatorname{Mor}(\mathcal{C})$ are morphisms $f: A \rightarrow B$ in $\mathcal{C}$, and a morphism between two objects $f: A \rightarrow B, f^{\prime}: A^{\prime} \rightarrow B^{\prime}$ of $\mathcal{M o r}(\mathcal{C})$ is a commutative diagram in $\mathcal{C}$

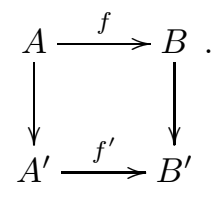

2.2. For any object $E \in D^{b}(X)$ and any subcategory $\mathcal{C}$ of $D^{b}(X)$, we will write $\operatorname{Hom}(\mathcal{C}, E)=0$ to mean $\operatorname{Hom}_{D^{b}(X)}(C, E)=0$ for all $C \in \mathcal{C}$, and similarly for $\operatorname{Hom}(E, \mathcal{C})=0$.

2.3. For any integer $d$, we will write $\operatorname{Coh}^{\leq d}(X)$ to denote the Serre subcategory of $\operatorname{Coh}(X)$ consisting of sheaves $E$ supported in dimension at most $d$. We will also write $\operatorname{Coh}^{\geq d}(X)$ to denote the full subcategory of $\operatorname{Coh}(X)$ consisting of sheaves $E$ such that $\operatorname{Hom}\left(\mathrm{Coh}^{\leq d-1}(X), E\right)=0$, i.e. sheaves $E$ that have no subsheaves supported in dimension $d-1$ or lower. Then we set $\operatorname{Coh}^{=d}(X)=\operatorname{Coh}^{\leq d}(X) \cap$ $\mathrm{Coh}^{\geq d}(X)$, which is the category of pure $d$-dimensional sheaves on $X$.

2.4. Given any object $E \in D^{b}(X)$ and any integer $i$, we will write $H^{i}(E)$ to denote the degree- $i$ cohomology of $E$ with respect to the standard t-structure on $D^{b}(X)$. We then define

$$
D_{\mathrm{Coh}(X)}^{\geq i}=\left\{E \in D^{b}(X): H^{k}(E)=0 \text { for all } k<i\right\}
$$

and similarly $D_{\operatorname{Coh}(X)}^{\leq i}$. For any integers $i \leq j$, we set

$$
D_{\operatorname{Coh}(X)}^{[i, j]}=D_{\operatorname{Coh}(X)}^{\geq i} \cap D_{\operatorname{Coh}(X)}^{\leq j} .
$$

2.5. Given a coherent sheaf $F$ on $X$, we will refer to the dimension (resp. codimension) of $\operatorname{supp}(F)$ simply as the dimension (resp. codimension) of $F$, and denote it as $\operatorname{dim} F$ (resp. $\operatorname{codim} F$ ).

2.6. For any $F \in D^{b}(X)$, we will write $F^{\vee}$ to denote the derived dual $R \mathscr{H}$ om $\left(F, \mathcal{O}_{X}\right)$ of $F$. When $F$ is a coherent sheaf of codimension $c$, we will write $F^{*}$ to denote the usual sheaf dual of $F$, i.e. $\mathcal{E} x t^{c}\left(F, \mathcal{O}_{X}\right)$; note that $H^{c}\left(F^{\vee}\right)=F^{*}$. Given a pure codimension-c coherent sheaf $F$ on $X$, we will say $F$ is reflexive if the natural injection $F \hookrightarrow F^{* *}$ is an isomorphism.

2.7. Recall that the homological dimension of a coherent sheaf $F$ on a smooth projective variety $X$ is defined to be the minimal length of a locally free resolution of $F$, and that a coherent sheaf of homological dimension $n$ satisfies $\mathcal{E} x t^{i}\left(F, \mathcal{O}_{X}\right)=0$ for all $i>n$ and hence $F^{\vee} \in D_{\operatorname{Coh}(X)}^{[0, n]}$. We will write $\mathrm{hd}(F)$ to denote the homological dimension of a coherent sheaf $F$ on $X$.

2.8. Stable pairs. On a smooth projective threefold $X$, a stable pair in the sense of PandharipandeThomas [13] is a pure 1-dimensional sheaf $F$ together with a section $\mathcal{O}_{X} \stackrel{s}{\rightarrow} F$ such that coker $(s)$ is 0 -dimensional. The purity of $F$ implies that the support of $F$ is a Cohen-Macaulay curve. We often think of a stable pair $\mathcal{O}_{X} \stackrel{s}{\rightarrow} F$ as a 2-term complex representing an object in $D^{b}(X)$, with $F$ sitting at degree 0 . We will refer to a stable pair in the sense of [13] as a PT stable pair, or simply a stable pair.

2.9. PT stable objects. Bayer characterised PT stable pairs using the notion of polynomial stability in [1]. There is a particular polynomial stability $\sigma_{P T}$ on $D^{b}(X)$, referred to as PT stability by Bayer, 
such that the $\sigma_{P T}$-stable objects objects $E$ in the heart

$$
\mathcal{A}^{p}:=\left\langle\mathrm{Coh}^{\geq 2}(X)[1], \operatorname{Coh}^{\leq 1}(X)\right\rangle
$$

with $\operatorname{ch}_{0}(E)=-1, \operatorname{ch}_{1}(E)=0$ and $\operatorname{det} E=\mathcal{O}_{X}$ are precisely the PT stable pairs in 2.8. We will refer to $\sigma_{P T}$-(semi)stable objects in $\mathcal{A}^{p}$ of any Chern character as PT (semi)stable objects. The properties of higher-rank PT stable objects and their moduli spaces were studied in [9, 11].

2.9.1. Suppose $X$ is a smooth projective threefold, and $\omega$ is a fixed ample class on $X$ that appears in the definition of PT stability. Then every PT semistable object $E$ with nonzero $\mathrm{ch}_{0}$ satisfies the following properties:

(i) $H^{-1}(E)$ is torsion-free and $\mu_{\omega}$-semistable,

(ii) $H^{0}(E)$ is 0-dimensional,

(iii) $\operatorname{Hom}_{D^{b}(X)}\left(\operatorname{Coh}^{\leq 0}(X), E\right)=0$;

moreover, when $\operatorname{ch}_{0}(E)$ and $\omega^{2} \operatorname{ch}_{1}(E)$ are coprime, every object in $\mathcal{A}^{p}$ satisfying (i) through (iii) is a PT stable object, and PT stability coincides with PT semistability [11, Proposition 2.24]. Also, properties (i) and (ii) implies that, if $E$ is a PT-semistable object, then $\operatorname{ch}_{0}(E)=-n$ for some nonnegative integer $n$; we will sometimes refer to such an $E$ as a rank $n$ PT semistable object by abuse of notation.

2.9.2. Under derived dual and up to a shift, $\mathrm{PT}$ stability corresponds to another polynomial stability $\sigma_{P T}^{*}$, meaning $\sigma_{P T}$-stable objects and $\sigma_{P T}^{*}$-stable objects correspond to each other via derived dual. We will refer to the $\sigma_{P T}^{*}$-(semi)stable objects as dual-PT (semi)stable objects; their properties and moduli spaces were studied in [10].

2.9.3. Suppose $X$ is a smooth projective threefold, and $\omega$ is a fixed ample class on $X$ that appears in the definition of dual-PT stability. Then a standard argument shows that every dual-PT semistable object $E$ with nonzero $\mathrm{ch}_{0}$ satisfies the following properties besides lying in $\mathcal{A}^{p}$ :

(i) $H^{-1}(E)$ is torsion-free and $\mu_{\omega}$-semistable.

(ii) $\operatorname{Hom}_{D^{b}(X)}\left(\mathrm{Coh}^{\leq 1}(X), E\right)=0$.

Property (ii) implies that $H^{-1}(E)$ is a reflexive sheaf. Also, when $\operatorname{ch}_{0}(E)$ and $\omega^{2} \operatorname{ch}_{1}(E)$ are coprime, every object in $\mathcal{A}^{p}$ satisfying (i) and (ii) is a dual-PT stable object, and dual-PT stability coincides with dual-PT semistability [10, Lemma 3.5].

Remark 2.10. In Toda's work [17], he directly defines PT semistable objects to be the objects in $D^{b}(X)$ satisfying properties (i) through (iii). All the computations in [17], however, are performed under the assumption that $\mathrm{ch}_{0}, \omega^{2} \mathrm{ch}_{1}$ are coprime; under this assumption, the PT semistable objects Toda studies coincide with the PT semistable objects defined using Bayer's polynomial stability (as in 2.9).

\section{THE DUALISING FUNCTOR}

In this section, we study the behaviour of a class of 2-term complexes under the derived dual functor ${ }^{\vee}$. These 2-term complexes can be taken to be various stable objects (see Section 5) and, in particular, PT stable objects.

Lemma 3.1. Let $E$ be an object of $D^{b}(X)$ satisfying $E^{\vee} \in D_{\operatorname{Coh}(X)}^{\leq 3}$. Then

$$
\operatorname{Hom}_{D^{b}(X)}\left(\operatorname{Coh}^{\leq 0}(X), E\right)=0 \text { if and only if } H^{3}\left(E^{\vee}\right)=0 \text {. }
$$

Proof. For any $E \in D^{b}(X)$ and $T \in \mathrm{Coh}^{\leq 0}(X)$ we have

$$
\operatorname{Hom}(T, E) \cong \operatorname{Hom}\left(E^{\vee}, T^{*}[-3]\right) .
$$


Therefore, when $E$ satisfies $E^{\vee} \in D_{\operatorname{Coh}(X)}^{\leq 3}$, we have $H^{3}\left(E^{\vee}\right)=0$ if and only if $\operatorname{Hom}(T, E)=0$ for all $T \in \operatorname{Coh}^{\leq 0}(X)$, i.e. $\operatorname{Hom}\left(\operatorname{Coh}^{\leq 0}(X), E\right)=0$.

Example 3.2. For any $E \in\left\langle\mathrm{Coh}^{\geq 1}(X)[1], \mathrm{Coh}^{\leq 1}(X)\right\rangle$, in the associated exact triangle

$$
H^{0}(E)^{\vee} \rightarrow E^{\vee} \rightarrow H^{-1}(E)^{\vee}[-1] \rightarrow H^{0}(E)^{\vee}[1]
$$

we have $H^{0}(E)^{\vee} \in D_{\operatorname{Coh}(X)}^{[2,3]}$ and $H^{-1}(E)^{\vee} \in D_{\operatorname{Coh}(X)}^{[0,2]}\left[8\right.$, Proposition 1.1.6] and hence $E^{\vee} \in D_{\operatorname{Coh}(X)}^{\leq 3}$. Thus

$$
\operatorname{Hom}\left(\operatorname{Coh}^{\leq 0}(X), E\right)=0 \text { if and only if } H^{3}\left(E^{\vee}\right)=0
$$

for such $E$ by Lemma 3.1 .

Lemma 3.3. Suppose $X$ is a smooth projective threefold and $F \in \mathrm{Coh}^{\geq 2}(X)$. Then

(i) F has homological dimension at most 1 if and only if

$$
\operatorname{Hom}\left(\operatorname{Coh}^{\leq 0}(X), F[1]\right)=0 .
$$

(ii) If $F$ is torsion-free, then $F$ is reflexive if and only if

$$
\operatorname{Hom}\left(\operatorname{Coh}^{\leq 1}(X), F[1]\right)=0 \text {. }
$$

Proof. (i) Taking derived dual, we observe that

$$
\operatorname{Hom}\left(\operatorname{Coh}^{\leq 0}(X), F[1]\right)=0 \quad \text { if and only if } \quad \operatorname{Hom}\left(F^{\vee}, \operatorname{Coh}^{\leq 0}(X)[-2]\right)=0
$$

for any $F \in D^{b}(X)$. For any $F \in \mathrm{Coh}^{\geq 2}(X)$, we know $F^{\vee} \in D_{\mathrm{Coh}(X)}^{[0,2]}$ from [8, Proposition 1.1.6], and so $F$ satisfies the equivalent conditions in (3.3.3) if and only if $F^{\vee} \in D_{\operatorname{Coh}(X)}^{[0,1]}$, which in turn is equivalent to $F$ having homological dimension at most 1 [6, III.6].

(ii) This is a special case of [3, Lemma 4.20].

The results in the remainder of this provide a common ground across the constructions in this article, Toda's work [17], Gholampour-Kool's work [5], and the author's previous work [10].

Lemma 3.4. The category

$$
\left\{E \in\left\langle\operatorname{Coh}^{=3}(X)[1], \operatorname{Coh}^{\leq 1}(X)\right\rangle: \operatorname{Hom}\left(\operatorname{Coh}^{\leq 0}(X), E\right)=0\right\}
$$

is invariant under the functor $\left({ }^{\vee}\right)[2]$. Moreover, $H^{-1}(E)$ is slope (semi-)stable if and only if $H^{-1}\left(E^{\vee}[2]\right)$ is so.

Proof. Take any object $E$ in the category (3.4.1). If $H^{-1}(E)=0$, then $E=H^{0}(E)$ is a pure 1dimensional sheaf, in which case $E^{\vee}[2] \cong \mathcal{E} x t^{2}\left(E, \mathcal{O}_{X}\right)$ is also a pure 1-dimensional sheaf and hence again lies in the category (3.4.1). So let us suppose $H^{-1}(E) \neq 0$ from now on. The exact triangle

$$
H^{0}(E)^{\vee} \rightarrow E^{\vee} \rightarrow H^{-1}(E)^{\vee}[-1] \rightarrow H^{0}(E)^{\vee}[1]
$$


gives the long exact sequence

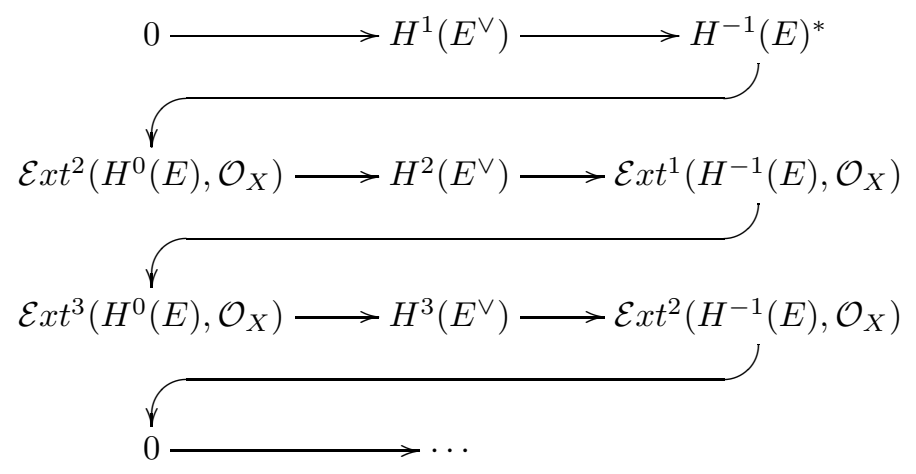

from which we see $H^{-1}\left(E^{\vee}[2]\right)=H^{1}\left(E^{\vee}\right)$ is a subsheaf of a torsion-free sheaf, and hence is torsionfree. We also have $H^{3}\left(E^{\vee}\right)=0$ by Example 3.2. Since $H^{-1}(E)$ is torsion-free, it follows that $\mathcal{E} x t^{1}\left(H^{-1}(E), \mathcal{O}_{X}\right) \in \operatorname{Coh}^{\leq 1}(X)$ [8, Proposition 1.1.6 ii)]. On the other hand, since $H^{0}(E) \in$ $\operatorname{Coh}^{\leq 1}(X)$, we have $\mathcal{E} x t^{2}\left(H^{0}(E), \mathcal{O}_{X}\right) \in \operatorname{Coh}^{\leq 1}(X)$. Hence $H^{2}\left(E^{\vee}\right)=H^{0}\left(E^{\vee}[2]\right) \in \operatorname{Coh}^{\leq 1}(X)$, giving us $E^{\vee}[2] \in\left\langle\mathrm{Coh}^{=3}(X)[1], \operatorname{Coh}^{\leq 1}(X)\right\rangle$ overall.

Let us write $F=E^{\vee}[2]$. Then $H^{3}\left(F^{\vee}\right)=H^{3}(E[-2])=0$, and so $\operatorname{Hom}\left(\operatorname{Coh}^{\leq 0}(X), F\right)=0$ by Example 3.2, i.e. $F$ lies in (3.4.1).

Lastly, if $H^{-1}(E)$ is slope (semi-)stable then so is its dual $H^{-1}(E)^{*}$; since $\mathcal{E} x t^{2}\left(H^{0}(E), \mathcal{O}_{X}\right)$ has codimension at least 2, this means that $H^{1}\left(E^{\vee}\right)=H^{-1}(F)$ is also slope (semi-)stable. On the other hand, if $H^{-1}(F)=H^{1}\left(E^{\vee}\right)$ is slope (semi-)stable, then so is $H^{-1}(E)^{*}$ from the long exact sequence above, implying $H^{-1}(E)^{* *}$ is also slope (semi-)stable. Since $H^{-1}(E)$ and $H^{-1}(E)^{* *}$ are isomorphic in codimension 1 , this means that $H^{-1}(E)$ itself is slope (semi-)stable.

3.5. The category $\mathrm{Coh}^{=1}(X)$ of pure 1-dimensional coherent sheaves on $X$ is invariant under the functor $\left(-^{\vee}\right)[2]$ by $[8, \mathrm{p} .6]$. As a result, for any $E \in D_{\operatorname{Coh}(X)}^{\leq 0}$ and $T \in \operatorname{Coh}^{=1}(X)$, we have the isomorphisms

$$
\operatorname{Hom}\left(T, E^{\vee}[2]\right) \cong \operatorname{Hom}\left(E, T^{\vee}[2]\right) \cong \operatorname{Hom}\left(E, T^{*}\right) \cong \operatorname{Hom}\left(H^{0}(E), T^{*}\right)
$$

where the first isomorphism follows from taking derived dual, and the last isomorphism uses the assumption $E \in D_{\mathrm{Coh}(X)}^{\leq 0}$.

Lemma 3.6. The functor $\left({ }^{\vee}\right)[2]$ induces an equivalence of subcategories of (3.4.1)

$$
\begin{aligned}
& \left\{E \in\left\langle\mathrm{Coh}^{=3}(X)[1], \mathrm{Coh}^{\leq 0}(X)\right\rangle: \operatorname{Hom}\left(\mathrm{Coh}^{\leq 0}(X), E\right)=0\right\} \stackrel{\sim}{\rightarrow} \\
& \left\{E \in\left\langle\operatorname{Coh}^{=3}(X)[1], \operatorname{Coh}^{\leq 1}(X)\right\rangle: \operatorname{Hom}\left(\mathrm{Coh}^{\leq 1}(X), E\right)=0\right\} .
\end{aligned}
$$

All the PT stable objects (resp. dual-PT stable objects) lie in the left-hand side (resp. right-hand side) of (3.6.1). As a result, Lemma 3.6 can be considered as a purely homological version of the statement that 'PT stable objects and dual-PT stable objects correspond to each other under derived dual'. We also note that this Lemma had essentially appeared in Piyaratne-Toda's work [14, Lemma 4.16] in their study of the moduli spaces of Bridgeland semistable objects on threefolds.

Also, by Lemma 3.3, all the torsion-free coherent sheaves of homological dimension at most 1 (resp. torsion-free reflexive sheaves) sitting at degree -1 lie in the left-hand side (resp. right-hand side) of (3.6.1).

Proof. Suppose $E$ is an object in the left-hand side of (3.6.1). By Lemma 3.4, it suffices to show that $\operatorname{Hom}\left(\operatorname{Coh}^{=1}(X), E^{\vee}[2]\right)=0$. For any $T \in \operatorname{Coh}^{=1}(X)$, we have $\operatorname{Hom}\left(T, E^{\vee}[2]\right) \cong \operatorname{Hom}\left(H^{0}(E), T^{*}\right)$ 
by (3.5.1); the latter Hom vanishes since $H^{0}(E) \in \mathrm{Coh}^{\leq 0}(X)$ by assumption while $T^{*}$ is pure 1dimensional.

Conversely, suppose $E$ is an object in the right-hand side of (3.6.1). Again, by Lemma 3.4, it suffices to show that $H^{0}\left(E^{\vee}[2]\right) \in \operatorname{Coh}^{\leq 0}(X)$. Since Lemma 3.4 already gives $H^{0}\left(E^{\vee}[2]\right) \in \operatorname{Coh}^{\leq 1}(X)$, it suffices to show $\operatorname{Hom}\left(H^{0}\left(E^{\vee}[2]\right), \operatorname{Coh}^{=1}(X)\right)=0$. To this end, take any $T \in \operatorname{Coh}^{=1}(X)$; note that $T$ is reflexive. Then (3.5.1) gives $\operatorname{Hom}\left(T^{*}, E\right) \cong \operatorname{Hom}\left(H^{0}\left(E^{\vee}[2]\right), T\right)$, in which the former Hom vanishes because $\operatorname{Hom}\left(\operatorname{Coh}^{\leq 1}(X), E\right)=0$ by assumption.

Corollary 3.7. The category

$$
\left\{E \in\left\langle\operatorname{Coh}^{=3}(X)[1], \operatorname{Coh}^{\leq 0}(X)\right\rangle: \operatorname{Hom}\left(\operatorname{Coh}^{\leq 1}(X), E\right)=0\right\}
$$

is invariant under the functor $-{ }^{\vee}[2]$.

Note that the category (3.7.1) contains all the reflexive sheaves (shifted by 1 ); in fact, it contains all the objects that are PT semistable and dual-PT semistable at the same time, the moduli space of which was studied in [10, Theorem 1.2] with a coprime assumption on the rank and degree of the objects.

\section{A FUNCTOR TAKING OBJECTS TO MORPHISMS}

In this section, we construct a functor $\widetilde{\mathcal{F}}$ that takes a subcategory $\mathcal{E}$ of $E \in D_{\operatorname{Coh}(X)}^{[-1,0]}$ into the category $\operatorname{Mor}\left(D^{b}(X)\right)$ (Proposition 4.4). Composing with the cohomology functor $H^{0}$, we obtain a functor from $\mathcal{E}$ into $\operatorname{Mor}(\operatorname{Coh}(X))$ (see 4.4.5).

4.1. We define the full subcategory of $D^{b}(X)$

$$
\mathcal{E}=\left\{E \in D_{\operatorname{Coh}(X)}^{[-1,0]}: \operatorname{hd}\left(H^{-1}(E)\right) \leq 1, H^{0}(E) \in \operatorname{Coh}^{\leq 0}(X)\right\}
$$

and the full subcategory of $\mathcal{M} \operatorname{or}\left(D^{b}(X)\right)$

$$
\mathcal{L}=\left\{A^{\vee}[1] \stackrel{m}{\rightarrow} B: A \in \operatorname{Coh}(X), \operatorname{hd}(A) \leq 1, B \in \operatorname{Coh}^{\leq 0}(X)\right\} .
$$

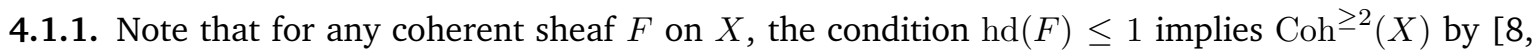
Proposition 1.1.6]. Hence $H^{-1}(E) \in \mathrm{Coh}^{\geq 2}(X)$ for any $E \in \mathcal{E}$.

4.2. For any $E \in \mathcal{E}$, truncation functors with respect to the standard t-structure on $D^{b}(X)$ give the canonical exact triangle

$$
H^{-1}(E)[1] \rightarrow E \rightarrow H^{0}(E) \stackrel{w}{\rightarrow} H^{-1}(E)[2] .
$$

Applying the derived dual functor to (4.2.1) followed by the shift functor [2] gives the exact triangle

$$
H^{0}(E)^{\vee}[2] \rightarrow E^{\vee}[2] \rightarrow H^{-1}(E)^{\vee}[1] \stackrel{w^{\vee}[3]}{\longrightarrow} H^{0}(E)^{\vee}[3]
$$

where $H^{-1}(E)^{\vee}[1] \in D_{\operatorname{Coh}(X)}^{[-1,0]}$ by the assumption $h d\left(H^{-1}(E)\right) \leq 1$, and $H^{0}(E)^{\vee}[3] \in \operatorname{Coh}^{\leq 0}(X)$. That is, the morphism $w^{\vee}[3]$ is an object of $\mathcal{L}$.

Definition 4.3. For any $E \in \mathcal{E}$, we define $\widetilde{\mathcal{F}}(E)$ to be the object $w^{\vee}[3]$ of $\mathcal{L}$ in the notation of 4.2 ,

Proposition 4.4. $\quad \widetilde{\mathcal{F}}$ is an essentially surjective (contravariant) functor from $\mathcal{E}$ to $\mathcal{L}$, and induces a bijection between the isomorphism classes in $\mathcal{E}$ and $\mathcal{L}$. 
Proof. Step 1. Given any morphism $E_{1} \stackrel{f}{\rightarrow} E_{2}$ in $\mathcal{E}$, the truncation functors give a morphism of exact triangles in $D^{b}(X)$

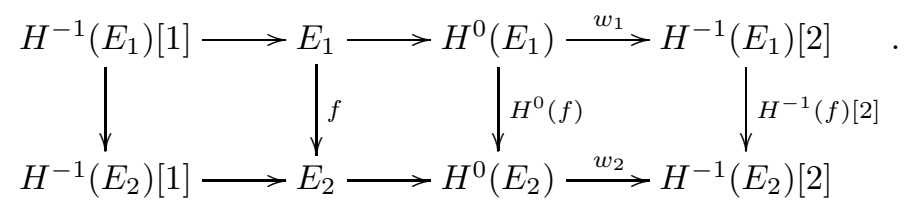

Applying the derived dual functor followed by the shift functor [2] gives

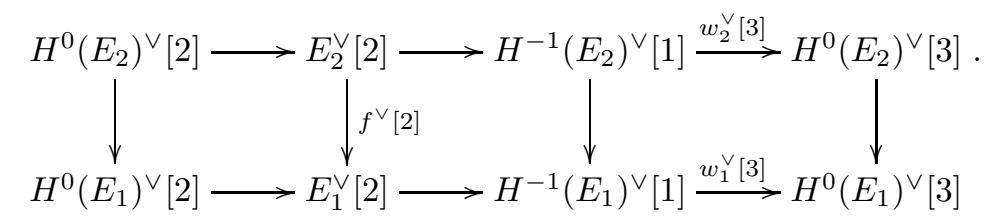

The right-most square now gives a morphism from $w_{1}^{\vee}[3]$ to $w_{2}^{\vee}[3]$ in $\mathcal{L}$. It is clear that $\widetilde{\mathcal{F}}$ respects composition of morphisms in $\mathcal{E}$, and so $\widetilde{\mathcal{F}}$ is a functor from $\mathcal{E}$ to $\mathcal{L}$.

Step 2. To show the essential surjectivity of $\widetilde{\mathcal{F}}$, let us take an arbitrary element of $\mathcal{L}$, say the diagram in $D^{b}(X)$

$$
A^{\vee}[1] \stackrel{m}{\rightarrow} B
$$

where $A$ is a sheaf of homological dimension at most 1 , and $B$ is a sheaf supported in dimension 0 . We first complete $m$ to an exact triangle in $D^{b}(X)$

$$
A^{\vee}[1] \stackrel{m}{\rightarrow} B \rightarrow C \rightarrow A^{\vee}[2] .
$$

Applying $[-3]^{\vee}$ now gives the exact triangle

$$
A[1] \rightarrow G \rightarrow B^{\vee}[3] \stackrel{m^{\vee}[3]}{\longrightarrow} A[2]
$$

where $G:=C^{\vee}[3]$ is an object of $\mathcal{E}$. Since $A[1] \in D_{\mathrm{Coh}(X)}^{\leq-1}$ and $B^{\vee}[3] \in D_{\operatorname{Coh}(X)}^{\geq 0}$, there is a canonical isomorphism of exact triangles in $D^{b}(X)$ [4, Lemma 5, IV.4]

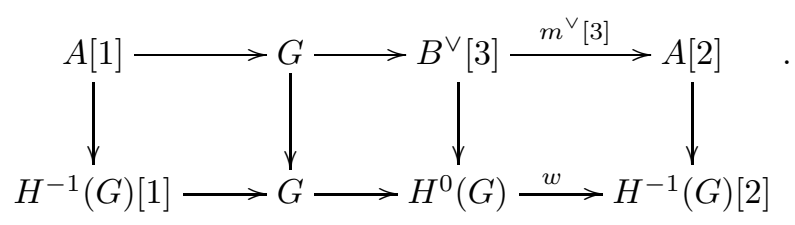

Applying the functor $\vee[2]$ gives the isomorphism of exact triangles

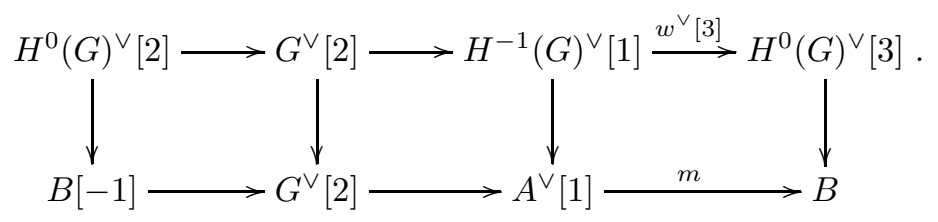

Since $w^{\vee}[3]$ is precisely $\widetilde{\mathcal{F}}(G)$, we have shown the essential surjectivity of $\widetilde{\mathcal{F}}$.

Step 3. To show that $\widetilde{\mathcal{F}}$ induces a bijection between the isomorphism classes in $\mathcal{E}$ and $\mathcal{L}$, let us take two objects $E_{1}, E_{2}$ in $\mathcal{E}$ and suppose there is an isomorphism from $\widetilde{\mathcal{F}}\left(E_{2}\right)$ to $\widetilde{\mathcal{F}}\left(E_{1}\right)$ in $\mathcal{L}$, say given by 
the diagram in $D^{b}(X)$

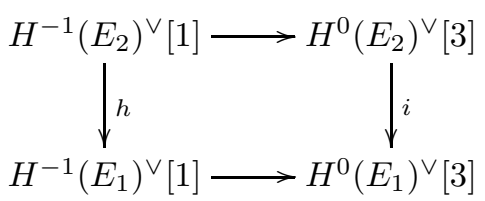

where $h, i$ are isomorphisms. We can complete the rows of this square to exact triangles of the form (4.2.2)

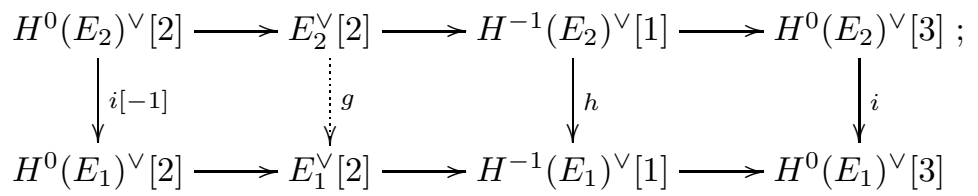

and then $h, i$ can be completed with an isomorphism $g: E_{2}^{\vee}[2] \rightarrow E_{1}^{\vee}[2]$ to an isomorphism of exact triangles [4, Corollary 4a, IV.1]. Hence $E_{1}$ and $E_{2}$ are isomorphic in $D^{b}(X)$. This completes the proof of the proposition.

4.4.4. In the proof of essential surjectivity in Proposition 4.4 (i.e. Step 2 of the proof), it is not clear that the construction taking the object $m$ in $\mathcal{L}$ to the object $G$ in $\mathcal{E}$ is a functor, since the object $C$ is defined up to an isomorphism that is not necessarily canonical.

4.4.5. The degree-zero cohomology functor with respect to the standard t-structure $H^{0}: D^{b}(X) \rightarrow$ $\operatorname{Coh}(X)$ induces a functor $\operatorname{Mor}\left(D^{b}(X)\right) \rightarrow \mathcal{M o r}(\operatorname{Coh}(X))$ which we will also denote by $H^{0}$. For any object in $\mathcal{L}$ of the form

$$
A^{\vee}[1] \stackrel{m}{\rightarrow} B
$$

its image under $H^{0}$ is

$$
H^{0}\left(A^{\vee}[1]\right)=\mathcal{E} x t^{1}\left(A, \mathcal{O}_{X}\right) \stackrel{H^{0}(m)}{\longrightarrow} B .
$$

Let us use the notation in the proof of Proposition 4.4 and take $C, w$ as in (4.4.1), (4.4.2), respectively. Then from (4.4.3), the morphism of sheaves $H^{0}(m)$ is surjective if and only if the morphism of sheaves $H^{0}\left(w^{\vee}[3]\right)$ is surjective.

\section{ESSENTIAL SURJECTIVITY OF THE FUNCTOR $\mathcal{F}$}

In this section, we will modify the functor $\widetilde{\mathcal{F}}$ to a new functor $\mathcal{F}$, and show that each object $E \in$ $D_{\mathrm{Coh}(X)}^{[-1,0]}$ of the following types is taken by $\mathcal{F}$ to a surjective morphism of sheaves $\mathcal{E} x t^{1}\left(H^{-1}(E), \mathcal{O}_{X}\right) \rightarrow$ $H^{0}(E)^{*}$ : PT-semistable objects, dual-PT semistable objects, objects giving rise to $L$-invariants in the sense of Toda [17], and stable frozen triples in the sense of Sheshmani [15]. In particular, under a coprime assumption on rank and degree, we prove in Theorem 5.9 that $\mathcal{F}$ restricts to an essentially surjective functor from the category of PT stable objects to a category of surjective morphisms of coherent sheaves.

Lemma 5.1. Suppose $E$ is an object in $\left\langle\mathrm{Coh}^{2}(X)[1], \mathrm{Coh}^{\leq 1}(X)\right\rangle$ and $H^{-1}(E)$ has homological dimension at most 1. Suppose

$$
H^{-1}(E)[1] \rightarrow E \rightarrow H^{0}(E) \stackrel{w}{\rightarrow} H^{-1}(E)[2]
$$

is the associated canonical exact triangle in $D^{b}(X)$. Then

$$
H^{0} \widetilde{\mathcal{F}}(E)=H^{0}\left(w^{\vee}[3]\right): \mathcal{E} x t^{1}\left(H^{-1}(E), \mathcal{O}_{X}\right) \rightarrow H^{0}(E)^{*}
$$

is a surjection in $\operatorname{Coh}(X)$ if and only if $\operatorname{Hom}_{D^{b}(X)}\left(\mathrm{Coh}^{\leq 0}(X), E\right)=0$. 
Proof. Applying $\vee[2]$ to (4.2.1) gives us the exact triangle

$$
H^{0}(E)^{\vee}[2] \rightarrow E^{\vee}[2] \rightarrow H^{-1}(E)^{\vee}[1] \stackrel{w^{\vee}[3]}{\longrightarrow} H^{0}(E)^{\vee}[3],
$$

which has long exact sequence of cohomology

$$
\cdots \rightarrow \mathcal{E} x t^{1}\left(H^{-1}(E), \mathcal{O}_{X}\right) \stackrel{H^{0}\left(w^{\vee}[3]\right)}{\longrightarrow} H^{0}(E)^{*} \rightarrow H^{1}\left(E^{\vee}[2]\right) \rightarrow \mathcal{E} x t^{2}\left(H^{-1}(E), \mathcal{O}_{X}\right)
$$

where the last term $\mathcal{E} x t^{2}\left(H^{-1}(E), \mathcal{O}_{X}\right)$ vanishes since $H^{-1}(E)$ has homological dimension at most 1 . Therefore, the morphism $H^{0} \widetilde{\mathcal{F}}(E)=H^{0}\left(w^{\vee}[3]\right)$ is surjective if and only if $H^{3}\left(E^{\vee}\right)=H^{1}\left(E^{\vee}[2]\right)=0$. On the other hand, $H^{3}\left(E^{\vee}\right)=0$ if and only if $\operatorname{Hom}\left(\operatorname{Coh}^{\leq 0}(X), E\right)=0$ by Example 3.2, and so we are done.

Example 5.2. Suppose $E$ is an object in $\left\langle\operatorname{Coh}^{=3}(X)[1], \operatorname{Coh}^{\leq 1}(X)\right\rangle$ that satisfies the vanishing

$$
\operatorname{Hom}\left(\operatorname{Coh}^{\leq 0}(X), E\right)=0 \text {. }
$$

Note that the category $\mathcal{A}:=\left\langle\mathrm{Coh}^{\geq 2}(X)[1], \mathrm{Coh}^{\leq 1}(X)\right\rangle$ is the heart of a t-structure on $D^{b}(X)$ and hence an abelian category [1, Section 3]. Also, the subcategory $\operatorname{Coh}^{\leq 0}(X)$ is closed under quotients in $\mathcal{A}$. Hence the vanishing $\operatorname{Hom}\left(\mathrm{Coh}^{\leq 0}(X), E\right)=0$ implies $\operatorname{Hom}\left(\mathrm{Coh}^{\leq 0}(X), H^{-1}(E)[1]\right)=0$, which in turn implies $H^{-1}(E)$ has homological dimension at most 1 by Lemma 3.3(i). That is, $E$ satisfies the hypotheses of Lemma 5.1. As a result, all of the following objects satisfy the hypotheses of Lemma 5.1 in addition to the vanishing $\operatorname{Hom}\left(\mathrm{Coh}^{\leq 0}(X),-\right)=0$ :

(a) Objects in the left-hand side of the equivalence (3.6.1), i.e. in the category

$$
\left\{E \in\left\langle\mathrm{Coh}^{=3}(X)[1], \operatorname{Coh}^{\leq 0}(X)\right\rangle: \operatorname{Hom}\left(\operatorname{Coh}^{\leq 0}(X), E\right)=0\right\} .
$$

These include all the PT-semistable objects (see 2.9.1).

(b) Objects in the right-hand side of the equivalence (3.6.1), i.e. in the category

$$
\left\{E \in\left\langle\operatorname{Coh}^{=3}(X)[1], \operatorname{Coh}^{\leq 1}(X)\right\rangle: \operatorname{Hom}\left(\operatorname{Coh}^{\leq 1}(X), E\right)=0\right\} .
$$

These include all the dual-PT semistable objects (see 2.9.3).

(c) Objects in

$$
\left\{E \in\left\langle\operatorname{Coh}_{\mu}(X), \mathcal{C}_{[0, \infty]}\right\rangle: \operatorname{Hom}\left(\mathcal{C}_{[0, \infty]}, E\right)=0\right\},
$$

which are the objects giving rise to the $L$-invariants defined by Toda in proving a higher-rank DT/PT correspondence in [17]. Here, we have some fixed ample class $\omega$ on $X$, and $\operatorname{Coh}_{\mu}(X)$ is the category of all $\mu_{\omega}$-semistable coherent sheaves $E$ with $\mu_{\omega}(E):=\omega^{2} \operatorname{ch}_{1}(E) / \operatorname{ch}_{0}(E)=\mu$. On the other hand, the category $\mathcal{C}_{[0, \infty]}$ consists of coherent sheaves $F$ supported in dimension at most 1, such that all its Harder-Narasimhan factors with respect to the slope function $\operatorname{ch}_{3}(-) / \omega \mathrm{ch}_{2}(-)$ have slopes lying in the interval $[0, \infty]$.

5.3. Lemma 5.1 motivates us to define the full subcategory of $\mathcal{E}$

$$
\mathcal{E}_{0}=\left\{E \in \mathcal{E}: \operatorname{Hom}_{D^{b}(X)}\left(\mathrm{Coh}^{\leq 0}(X), E\right)=0\right\} .
$$

For any coherent sheaf $F$ on $X$, we will also define the full subcategory of $\operatorname{Mor}(\operatorname{Coh}(X))$

$$
\mathcal{S}(F)=\left\{F \stackrel{q}{\rightarrow} Q: q \text { is a surjection in } \operatorname{Coh}(X), Q \in \mathrm{Coh}^{\leq 0}(X)\right\} .
$$

For any $E \in \mathcal{E}_{0}$, we have $H^{-1}(E) \in \mathrm{Coh}^{\geq 2}(X)$ by 4.1.1, and so $E$ satisfies the hypotheses of Lemma 5.1. Since $H^{0}(E)$ is 0-dimensional, we obtain that $H^{0} \widetilde{\mathcal{F}}(E)$ lies in the category $\mathcal{S}\left(\mathcal{E} x t^{1}\left(H^{-1}(E), \mathcal{O}_{X}\right)\right)$. Note that $H^{-1}(E)$ has homological dimension at most 1 from the definition of $\mathcal{E}$. This allows us to make the following definition:

Definition 5.4. We write $\mathcal{F}$ to denote the restriction of $\widetilde{\mathcal{F}}$

$$
\mathcal{F}=\left.\left(H^{0} \circ \widetilde{\mathcal{F}}\right)\right|_{\mathcal{E}_{0}}: \mathcal{E}_{0} \rightarrow \coprod_{F \in \operatorname{Coh}(X), \operatorname{hd}(F) \leq 1} \mathcal{S}\left(\mathcal{E} x t^{1}\left(F, \mathcal{O}_{X}\right)\right) .
$$


Example 5.5. (PT semistable objects) Every PT semistable object $E$ of nonzero $\mathrm{ch}_{0}$ lies in $\mathcal{E}_{0}$. To see this, note that the canonical exact triangle $H^{-1}(E)[1] \rightarrow E \rightarrow H^{0}(E) \rightarrow H^{-1}(E)[2]$ gives a short exact sequence $0 \rightarrow H^{-1}(E)[1] \rightarrow E \rightarrow H^{0}(E) \rightarrow 0$ in $\mathcal{A}^{p}$. Since $\mathrm{Coh}^{\leq 0}(X)$ is closed under quotient in $\mathcal{A}^{p}$, the vanishing $\operatorname{Hom}\left(\mathrm{Coh}^{\leq 0}(X), E\right)=0$ implies the vanishing $\operatorname{Hom}\left(\operatorname{Coh}^{\leq 0}(X), H^{-1}(E)[1]\right)=0$, and so $\mathrm{hd}\left(H^{-1}(E)\right) \leq 1$ by Lemma 3.3(i). The claim then follows from 2.9.1

Example 5.6. (Sheshmani's frozen triples) Every stable frozen triple on a smooth projective threefold $X$ in the sense of Sheshmani [15] represents an object in $\mathcal{E}_{0}$. A frozen triple $(G, F, \varphi)$ consists of a locally free sheaf $G \cong \mathcal{O}_{X}^{\oplus r}$ on $X$ (for some positive integer $r$ ) together with a morphism of coherent sheaves $\varphi: G \rightarrow F$ where $F$ is pure 1-dimensional. Such a frozen triple is called stable (or $\tau^{\prime}$-stable in [15]) if the cokernel of $\varphi$ is 0-dimensional. Let us write $E$ to represent the 2-term complex $[G \stackrel{\varphi}{\rightarrow} F]$ in $D^{b}(X)$ with $F$ sitting at degree 0 . Then clearly $E \in \mathcal{A}^{p}$. For any $T \in \operatorname{Coh}^{\leq 0}(X)$, applying $\operatorname{Hom}(T,-)$ to the exact triangle in $D^{b}(X)$

$$
G \stackrel{\varphi}{\rightarrow} F \rightarrow E \rightarrow G[1]
$$

gives $\operatorname{Hom}(T, E)=0$ and so $\operatorname{Hom}\left(\operatorname{Coh}^{\leq 0}(X), E\right)=0$. The same argument as in Example 5.5 then shows hd $\left(H^{-1}(E)\right) \leq 1$, and so $E \in \mathcal{E}_{0}$. Note that this example is already implicitly stated in [17, Example 3.2].

Lemma 5.7. Given any $A \in \operatorname{Coh}(X)$ with $\mathrm{hd}(A) \leq 1$ and a morphism of sheaves $r: \mathcal{E} x t^{1}\left(A, \mathcal{O}_{X}\right) \rightarrow$ $Q$ where $Q \in \operatorname{Coh}^{\leq 0}(X)$, there exists an object $G$ in $\mathcal{E}$ such that $H^{0} \widetilde{\mathcal{F}}(G)$ is isomorphic to $r$ in $\mathcal{M}$ or $(\operatorname{Coh}(X))$. Moreover, if $r$ is a surjection in $\operatorname{Coh}(X)$, then we can take $G$ to be in $\mathcal{E}_{0}$.

Proof. Consider the composition of morphisms in $D^{b}(X)$

$$
A^{\vee}[1] \stackrel{c}{\rightarrow} H^{0}\left(A^{\vee}[1]\right)=\mathcal{E} x t^{1}\left(A, \mathcal{O}_{X}\right) \stackrel{r}{\rightarrow} Q
$$

where $c$ is canonical. Since $r c \in \mathcal{L}$, the essential surjectivity of $\widetilde{\mathcal{F}}$ from Proposition 4.4 implies that there exists some $G \in \mathcal{E}$ such that $\widetilde{\mathcal{F}}(G) \cong r c$ in $\mathcal{L}$, which in turn implies $r \cong H^{0} \widetilde{\mathcal{F}}(G)$ in $\operatorname{Mor}(\operatorname{Coh}(X))$.

In fact, from the construction of the object $G$ (see Step 2 of the proof of Proposition 4.4), we have the exact triangle in $D^{b}(X)$

$$
A[1] \rightarrow G \rightarrow Q^{\vee}[3] \rightarrow A[2]
$$

which gives $H^{-1}(G) \cong A$ and $H^{0}(G) \cong Q^{*}$, i.e. $G$ satisfies the hypotheses of Lemma 5.1. Therefore, if $r$ is a surjection in $\operatorname{Coh}(X)$, the morphism of sheaves $H^{0} \widetilde{\mathcal{F}}(G)$ is also a surjection in $\operatorname{Coh}(X)$, and Lemma 5.1]implies that $\operatorname{Hom}\left(\mathrm{Coh}^{\leq 0}(X), G\right)=0$, meaning $G \in \mathcal{E}_{0}$.

5.8. We have the following restriction of $\mathcal{F}$ :

$$
\begin{aligned}
\mathcal{F}_{\mathrm{tf}}:\left\{E \in \mathcal{E}_{0}: H^{-1}(E) \text { is torsion free }\right\} & \rightarrow \coprod_{A \in \mathrm{Coh}^{=3}(X), \operatorname{hd}(A) \leq 1} \mathcal{S}\left(\mathcal{E} x t^{1}\left(A, \mathcal{O}_{X}\right)\right) \\
E & \mapsto \mathcal{F}(E) .
\end{aligned}
$$

If we fix an ample divisor $\omega$ on $X$ and $r \in H^{0}(X), D \in H^{2}(X)$ such that $r, \omega^{2} D$ are coprime, then from 2.9.1 we know that an object $E$ with $\operatorname{ch}_{0}(E)=-r$ and $\operatorname{ch}_{1}(E)=-D$ lies in $\mathcal{E}_{0}$ if and only if it is a PT stable object. Let us write

$$
\mathcal{H}=\left\{A \in \operatorname{Coh}^{=3}(X): \operatorname{hd}(A) \leq 1, A \text { is } \mu_{\omega} \text {-stable }\right\} .
$$


Then we can further restrict $\mathcal{F}$ to:

$$
\begin{aligned}
\mathcal{F}_{P T, r, D}:\left\{E \text { is a PT stable object : } \operatorname{ch}_{0}(E)=-r, \operatorname{ch}_{1}(E)=-D\right\} & \rightarrow \coprod_{\substack{A \in \mathcal{H} \\
\operatorname{ch}_{0}(A)=r, \operatorname{ch}_{1}(A)=D}} \mathcal{S}\left(\mathcal{E} x t^{1}\left(A, \mathcal{O}_{X}\right)\right) \\
E & \mapsto \mathcal{F}(E)
\end{aligned}
$$

Theorem 5.9. The functors $\mathcal{F}, \mathcal{F}_{\mathrm{tf}}$ are essentially surjective. If $\omega$ is an ample divisor on $X$ and $r \in$ $H^{0}(X), D \in H^{2}(X)$ are such that $r, \omega^{2} D$ are coprime, then $\mathcal{F}_{P T, r, D}$ is also essentially surjective.

Proof. The essential surjectivity of $\mathcal{F}$ follows from Lemma 5.7, while that of $\mathcal{F}_{\mathrm{tf}}, \mathcal{F}_{P T, r, D}$ follows from the essential surjectivity of $\mathcal{F}$ itself and the discussion in 5.8.

\section{FIBERS OF THE FUNCTOR $\mathcal{F}$}

Given an object $E$ of $\mathcal{E}_{0}$, the functor $\mathcal{F}$ constructed in Section 5 takes $E$ to a surjective morphism of coherent sheaves

$$
\mathcal{F}(E): \mathcal{E} x t^{1}\left(H^{-1}(E), \mathcal{O}_{X}\right) \rightarrow H^{0}(E)^{*}
$$

In this section, we answer the following questions:

(1) Given an object $E \in \mathcal{E}_{0}$, how do we enumerate all the objects $\bar{E} \in \mathcal{E}_{0}$ such that $\mathcal{F}(E)$ and $\mathcal{F}(\bar{E})$ are isomorphic in $\mathcal{M o r}(\operatorname{Coh}(X))$ ?

(2) Given two objects $E, \bar{E}$ of $\mathcal{E}_{0}$ such that $\mathcal{F}(E), \mathcal{F}(\bar{E})$ are isomorphic in $\mathcal{M o r}(\operatorname{Coh}(X))$, precisely when are $E, \bar{E}$ isomorphic in $\mathcal{E}_{0}$ ?

These questions are answered in Lemma 6.2 and Lemma 6.3, respectively.

For the purpose of computing invariants, however, it may help to think of $\mathcal{F}(\bar{E})$ as a point of a quot scheme.

Definition 6.1. For any coherent sheaf $F$ on $X$, we will write $\mathcal{Q}(F)$ to denote the subcategory of $\operatorname{Mor}(\operatorname{Coh}(X))$ where the set of objects is

$$
\mathcal{Q}(F)=\left\{F \stackrel{q}{\rightarrow} Q: q \text { is a surjection in } \operatorname{Coh}(X), Q \in \mathrm{Coh}^{\leq 0}(X)\right\},
$$

and where the morphisms from an object $F \stackrel{q_{1}}{\longrightarrow} Q_{1}$ to another $F \stackrel{q_{2}}{\longrightarrow} Q_{2}$ are commutative diagrams in $\operatorname{Coh}(X)$ of the form

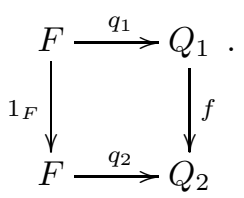

A morphism in $\mathcal{Q}(F)$ as above is an isomorphism if and only if $f$ is an isomorphism in $\operatorname{Coh}(X)$. Note that $\mathcal{Q}(F)$ is not a full subcategory of $\mathcal{M o r}(\operatorname{Coh}(X))$, i.e. $\mathcal{Q}(F)$ has 'fewer' arrows than $\mathcal{M o r}(\operatorname{Coh}(X))$.

If we have an isomorphism $E \cong \bar{E}$ in $\mathcal{E}_{0}$, then this isomorphism induces an isomorphism $\mathcal{F}(E) \cong$ $\mathcal{F}(\bar{E})$ in $\operatorname{Mor}(\operatorname{Coh}(X))$ by virtue of $\mathcal{F}$ being a functor. When $H^{-1}(E)=H^{-1}(\bar{E})$, however, it is not necessarily the case that an isomorphism $E \cong \bar{E}$ in $\mathcal{E}_{0}$ induces an isomorphism in the category $\mathcal{Q}\left(\mathcal{E} x t^{1}\left(H^{-1}(E), \mathcal{O}_{X}\right)\right)$. If $E, \bar{E} \in \mathcal{E}_{0}$ satisfy $H^{-1}(E)=H^{-1}(\bar{E})$ and $\mathcal{F}(E), \mathcal{F}(\bar{E})$ are isomorphic as objects of $\mathcal{Q}\left(\mathcal{E} x t^{1}\left(H^{-1}(E), \mathcal{O}_{X}\right)\right)$, however, it is indeed true that $E$ and $\bar{E}$ are isomorphic in $D^{b}(X)$ by 6.3.3. We phrase this formally in Lemma 6.5.

In the last part of this section, we revisit a construction mentioned in [5, p.3] in which a surjection of sheaves $\mathcal{E} x t^{1}\left(I_{C}, \mathcal{O}_{X}\right) \rightarrow Q$, where $I_{C}$ is the ideal sheaf of a Cohen-Macaulay curve $C$ on $X$ and $Q$ is a 0-dimensional sheaf, gives rise to a PT stable pair (i.e. a rank-one PT stable object). We generalise 
this construction to higher ranks, and compare the generalisation with the functor $\mathcal{F}$ constructed in Section 5 ,

Lemma 6.2. Fix an element $\bar{E} \in \mathcal{E}_{0}$. The functor

$$
\begin{aligned}
\mathcal{G}:\left\{E \in \mathcal{E}_{0}: \mathcal{F}(E) \cong \mathcal{F}(\bar{E})\right\} & \rightarrow\left\{A \in \operatorname{Coh}(X): \operatorname{hd}(A) \leq 1, \mathcal{E} x t^{1}\left(A, \mathcal{O}_{X}\right) \cong \mathcal{E} x t^{1}\left(H^{-1}(\bar{E}), \mathcal{O}_{X}\right)\right\} \\
E & \mapsto H^{-1}(E)
\end{aligned}
$$

is essentially surjective.

Proof. That $\mathcal{G}$ is a functor from the stated domain to the stated codomain follows from the definitions of $\mathcal{E}$ and the construction of the functor $\mathcal{F}$. To see the essential surjectivity of $\mathcal{G}$, take any $A$ in the codomain of $\mathcal{G}$ and fix an isomorphism of sheaves $\alpha: \mathcal{E} x t^{1}\left(A, \mathcal{O}_{X}\right) \rightarrow \mathcal{E} x t^{1}\left(H^{-1}(\bar{E}), \mathcal{O}_{X}\right)$. Let $c$ denote the canonical map $A^{\vee}[1] \rightarrow H^{0}\left(A^{\vee}[1]\right)$. The composite map

$$
A^{\vee}[1] \stackrel{c}{\rightarrow} H^{0}\left(A^{\vee}[1]\right) \stackrel{\alpha}{\rightarrow} H^{0}\left(H^{-1}(\bar{E})^{\vee}[1]\right) \stackrel{\mathcal{F}(\bar{E})}{\rightarrow} H^{0}(\bar{E})^{\vee}[3]
$$

can be completed to an exact triangle in $D^{b}(X)$

$$
A^{\vee}[1] \stackrel{\mathcal{F}(\bar{E}) \circ \alpha \circ c}{\longrightarrow} H^{0}(\bar{E})^{\vee}[3] \rightarrow G^{\vee}[3] \rightarrow A^{\vee}[2]
$$

for some object $G$ (which is unique up to a non-canonical isomorphism [7, TR3, 1.2]). Applying $-{ }^{\vee}[3]$ gives us the second row of the following diagram; the first row is constructed using truncation functors, while the unmarked vertical maps are canonical and induced by the identity map on $G$, 4 , IV.4 Lemma 5b]:

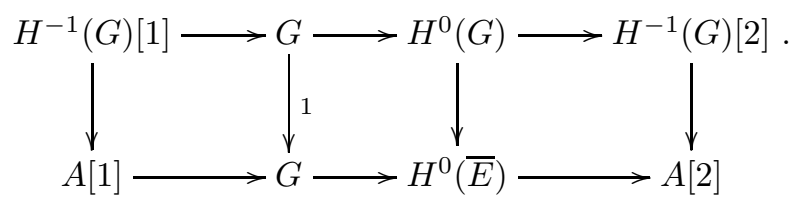

Applying $-{ }^{\vee}[2]$ to the entire diagram above now gives the isomorphism of exact triangles

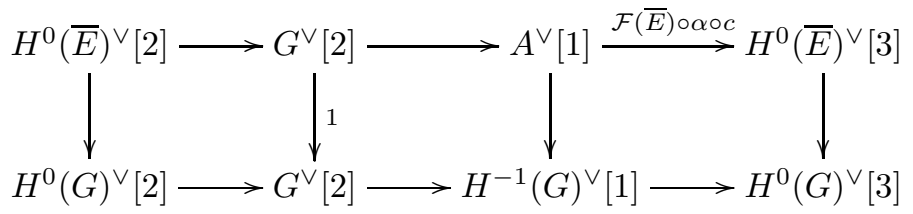

in which the right-most square factorises as

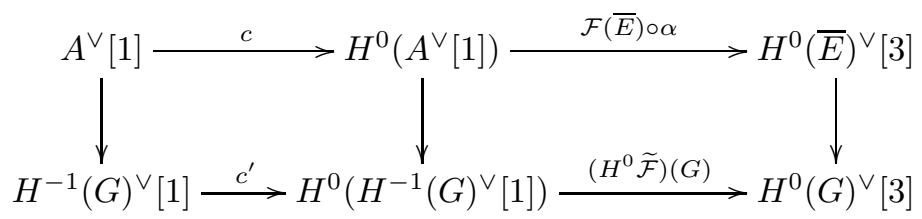

through canonical maps $c, c^{\prime}$.

Since $\bar{E} \in \mathcal{E}_{0}$ by assumption, the morphism of sheaves $\mathcal{F}(\bar{E})$, and hence $\mathcal{F}(\bar{E}) \circ \alpha$, is surjective. It follows that $\left(H^{0} \widetilde{\mathcal{F}}\right)(G)$ is also surjective by the commutativity of the right-hand square of $(6.2 .2)$, and so $G \in \mathcal{E}_{0}$ by Lemma 5.1. Hence we can write $\left(H^{0} \widetilde{\mathcal{F}}\right)(G)$ as $\mathcal{F}(G)$.

Note that in (6.2.2), the map $c^{\prime}$ is canonical and all the vertical maps are isomorphisms. Taking inverses of the vertical maps in the right-hand square in (6.2.2), the following concatenation gives an 
isomorphism $\mathcal{F}(G) \rightarrow \mathcal{F}(\bar{E})$ :

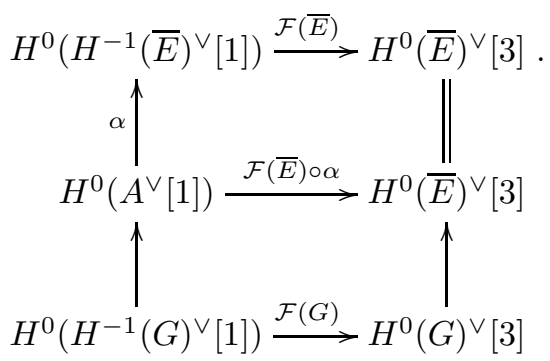

That is, $G$ is an object in the domain of the functor $\mathcal{G}$ such that $\mathcal{G}(G)=H^{-1}(G) \cong A$ (this isomorphism follows from the left-most vertical map in (6.2.2) ), proving the essential surjectivity of $\mathcal{G}$.

6.2.3. Given a fixed object $\bar{E} \in \mathcal{E}_{0}$, the proof of Lemma 6.2 says we can construct all the objects in $\left\{E \in \mathcal{E}_{0}: \mathcal{F}(E) \cong \mathcal{F}(\bar{E})\right\}$ by first going through all the coherent sheaves $A$ of homological dimension at most 1 for which there exists a sheaf isomorphism $\alpha: \mathcal{E} x t^{1}\left(A, \mathcal{O}_{X}\right) \rightarrow \mathcal{E} x t^{1}\left(H^{-1}(\bar{E}), \mathcal{O}_{X}\right)$, and then completing composite maps (6.2.1) to exact triangles.

For objects $E, \bar{E} \in \mathcal{E}_{0}$, the following lemma gives a comparison between the condition of $E, \bar{E}$ being isomorphic in $D^{b}(X)$ and the condition of $\mathcal{F}(E), \mathcal{F}(\bar{E})$ being isomorphic in $\operatorname{Mor}(\operatorname{Coh}(X))$.

Lemma 6.3. Given $E, \bar{E} \in \mathcal{E}_{0}$, the following are equivalent:

(i) $E, \bar{E}$ are isomorphic in $D^{b}(X)$.

(ii) There exists an isomorphism $\mathcal{F}(E) \rightarrow \mathcal{F}(\bar{E})$ in $\operatorname{Mor}(\operatorname{Coh}(X))$

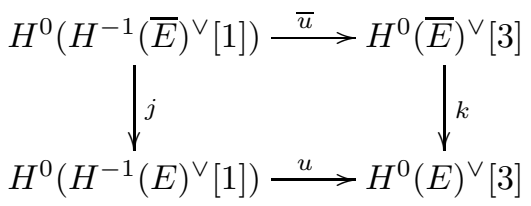

and an isomorphism $H^{-1}(\bar{E})^{\vee}[1] \stackrel{i}{\rightarrow} H^{-1}(E)^{\vee}[1]$ in $D^{b}(X)$ such that $H^{0}(i)=j$.

Proof. The implication (i) $\Rightarrow$ (ii) follows from the construction of the functor $\mathcal{F}$.

Let us now assume (ii) holds. We can concatenate (6.3.1) with the commutative square induced by $i$ to form

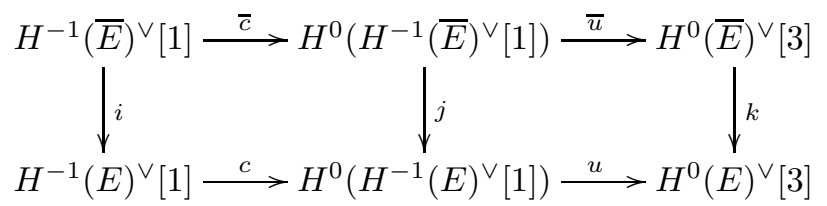

where $\bar{c}, c$ are canonical maps. Since $\mathcal{F}(E)=u$ and $\mathcal{F}(\bar{E})=\bar{u}$ in our notation, from the construction of $\mathcal{F}$ we know the composite maps $\bar{u} \bar{c}, u c$ can be completed to exact triangles with $\bar{E}^{\vee}[3], E^{\vee}[3]$ :

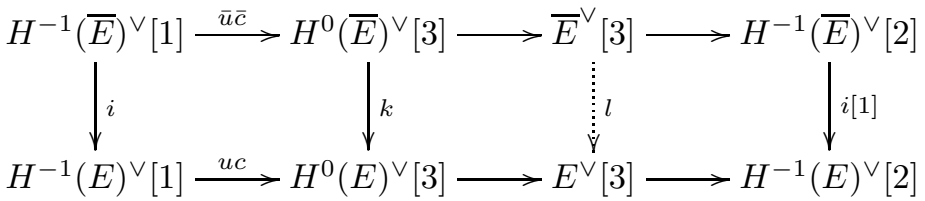

and then $i, k$ induce an isomorphism $l$ in $D^{b}(X)$, and so (i) holds. 
6.3.3. Suppose $E, \bar{E} \in \mathcal{E}_{0}$ satisfy $H^{-1}(E)=H^{-1}(\bar{E})$ and $\mathcal{F}(E), \mathcal{F}(\bar{E})$ are isomorphic in $\mathcal{Q}\left(H^{-1}(E)\right)$. Then by taking $i=1_{H^{-1}(E)^{\vee}[1]}$ in the proof of Lemma 6.3, we see that $E, \bar{E}$ are isomorphic in $D^{b}(X)$. Let us phrase this in a slightly more formal framework in Lemma 6.5 below.

6.4. Let us define a subcategory $\mathcal{E}_{0}^{\prime}$ of $\mathcal{E}_{0}$ where the objects of $\mathcal{E}_{0}^{\prime}$ are the same as those of $\mathcal{E}_{0}$, but where a morphism $E \stackrel{f}{\rightarrow} \bar{E}$ in $\mathcal{E}_{0}^{\prime}$ is a morphism in $\mathcal{E}_{0}$ with the extra requirement that, in the induced morphism of exact triangles

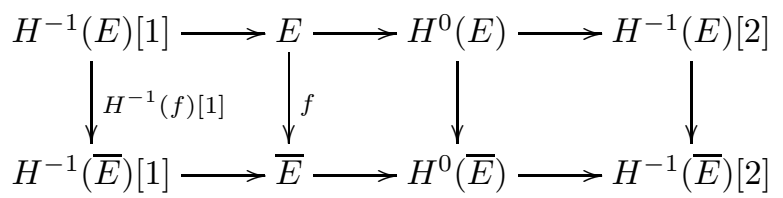

we must have

$$
H^{-1}(f)=1_{H^{-1}(E)} \text {. }
$$

As a result, if $E, \bar{E}$ are two objects of $\mathcal{E}_{0}$ such that $H^{-1}(E) \neq H^{-1}(\bar{E})$, then $\operatorname{Hom}_{\mathcal{E}_{0}^{\prime}}(E, \bar{E})=\varnothing$, meaning $\mathcal{E}_{0}^{\prime}$ is a non-full subcategory of $\mathcal{E}_{0}$. It is easy to see that $\mathcal{F}$ restricts to a functor

$$
\mathcal{F}^{\prime}: \mathcal{E}_{0}^{\prime} \rightarrow \coprod_{F \in \operatorname{Coh}(X), \operatorname{hd}(F) \leq 1} \mathcal{Q}\left(\mathcal{E} x t^{1}\left(F, \mathcal{O}_{X}\right)\right)
$$

For a fixed object $\bar{E}$ of $\mathcal{E}_{0}^{\prime}$, we also define the full subcategory of $\mathcal{E}_{0}^{\prime}$

$$
\mathcal{E}_{0}^{\prime}(\bar{E}):=\left\{E \in \mathcal{E}_{0}^{\prime}: H^{-1}(E)=H^{-1}(\bar{E}), \mathcal{F}^{\prime}(E) \cong \mathcal{F}^{\prime}(\bar{E}) \text { in } \mathcal{Q}\left(\mathcal{E} x t^{1}\left(H^{-1}(\bar{E}), \mathcal{O}_{X}\right)\right)\right\} .
$$

Lemma 6.5. Let $\bar{E}$ be a fixed object of $\mathcal{E}_{0}^{\prime}$. Then $\mathcal{F}^{\prime}$ further restricts to a functor

$$
\mathcal{E}_{0}^{\prime}(\bar{E}) \rightarrow \mathcal{Q}\left(\mathcal{E} x t^{1}\left(H^{-1}(\bar{E}), \mathcal{O}_{X}\right)\right)
$$

which induces an injection from the set of isomorphism classes in the domain to the set of isomorphism classes in the codomain.

Proof. Suppose $E, \bar{E}$ are two objects of $\mathcal{E}_{0}^{\prime}$ such that $H^{-1}(E)=H^{-1}(\bar{E})$ and $\mathcal{F}^{\prime}(E) \cong \mathcal{F}^{\prime}(\bar{E})$ in the category $\mathcal{Q}\left(\mathcal{E} x t^{1}\left(H^{-1}(\bar{E}), \mathcal{O}_{X}\right)\right)$. Then we have a commutative diagram in $D^{b}(X)$

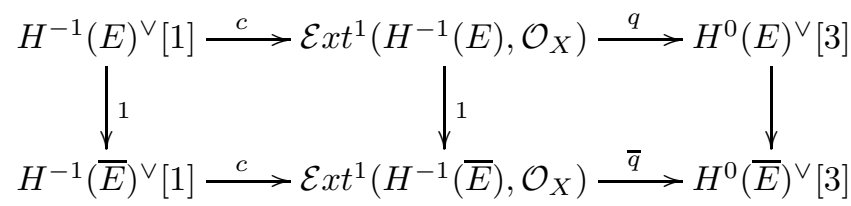

where $c$ is the canonical map, the right-hand vertical arrow is an isomorphism, and $q=\mathcal{F}^{\prime}(E), \bar{q}=$ $\mathcal{F}^{\prime}(\bar{E})$. From the construction of the functor $\mathcal{F}$, we can complete $q c, \bar{q} c$ to exact triangles with $E^{\vee}[2], \bar{E}^{\vee}[2]$ and obtain an isomorphism of exact triangles

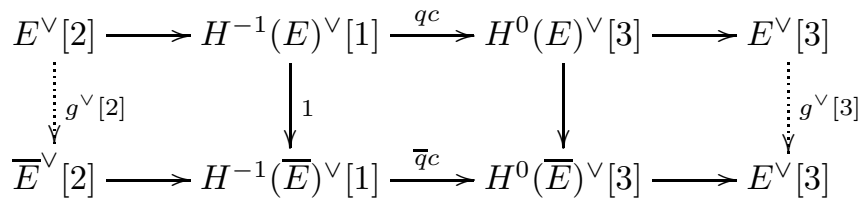

for some isomorphism $\bar{E} \stackrel{g}{\rightarrow} E$ in $D^{b}(X)$. Dualising, we obtain the isomorphism of triangles

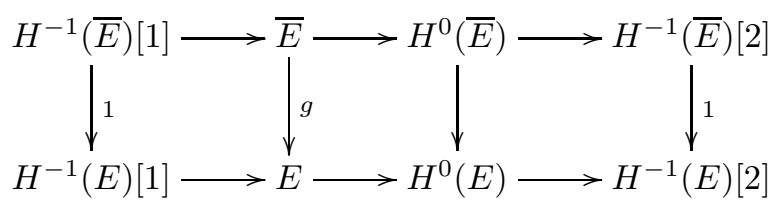


from which we see $H^{-1}(g)=1_{H^{-1}(\bar{E})}$ by [4, IV.4 Lemma 5b)]. That is, the morphism $g$ is a morphism in the category $\mathcal{E}_{0}^{\prime}$.

Lemma 6.6. We have

$$
\left\{E \in \mathcal{E}_{0}: \operatorname{ch}_{0}(E)=-1, \operatorname{ch}_{1}(E)=0, H^{-1}(E) \text { is torsion-free }\right\}=\{\text { rank-one PT stable objects }\} .
$$

Proof. The inclusion from left to right follows from 2.9.1. To see the other inclusion, take any rankone PT stable object $E$. Then $E \in \mathcal{A}^{p}$, and the canonical exact triangle (4.2.1) makes $H^{-1}(E)[1]$ an $\mathcal{A}^{p}$-subobject of $E$. Since $\operatorname{Coh}^{\leq 0}(X)$ is closed under quotient in $\mathcal{A}^{p}$ and we have the vanishing $\operatorname{Hom}\left(\operatorname{Coh}^{\leq 0}(X), E\right)=0$ from the PT stability of $E$, it follows that $\operatorname{Hom}\left(\operatorname{Coh}^{\leq 0}(X), H^{-1}(E)[1]\right)=0$. Lemma 3.3 then implies hd $\left(H^{-1}(E)\right) \leq 1$, and so $E$ lies in the category on the left-hand side.

6.7. For a fixed Cohen-Macaulay curve $C$ on a smooth projective threefold $X$, Gholampour-Kool describes a construction that takes an element of $\mathcal{Q}\left(\mathcal{E} x t^{1}\left(I_{C}, \mathcal{O}_{X}\right)\right)$ to a stable pair on $X$ [5, p.3]. This construction is as follows: given a surjection $q: \mathcal{E} x t^{1}\left(I_{C}, \mathcal{O}_{X}\right) \rightarrow Q$ in $\operatorname{Coh}(X)$ where $Q$ is 0 -dimensional, let $K=\operatorname{ker}(q)$ so that we have a short exact sequence of sheaves

$$
0 \rightarrow K \rightarrow \mathcal{E} x t^{1}\left(I_{C}, \mathcal{O}_{X}\right) \stackrel{q}{\rightarrow} Q \rightarrow 0 .
$$

Taking derived dual and then taking cohomology, and noting that $\mathcal{E} x t^{1}\left(I_{C}, \mathcal{O}_{X}\right) \cong \mathcal{O}_{C}^{*}$ where $\mathcal{O}_{C}$ is reflexive, we obtain the short exact sequence

$$
0 \rightarrow \mathcal{O}_{C} \rightarrow K^{*} \rightarrow Q^{*} \rightarrow 0 .
$$

Taking the Yoneda product of the last exact sequence with the structural exact sequence

$$
0 \rightarrow I_{C} \rightarrow \mathcal{O}_{X} \rightarrow \mathcal{O}_{C} \rightarrow 0
$$

then gives the four-term exact sequence

$$
0 \rightarrow I_{C} \rightarrow \mathcal{O}_{X} \stackrel{s}{\rightarrow} K^{*} \rightarrow Q^{*} \rightarrow 0
$$

and hence a stable pair

$$
\mathcal{O}_{X} \stackrel{s}{\rightarrow} F
$$

where $F:=K^{*}$.

6.8. The construction in 6.7 can be generalised to higher ranks. This is already mentioned in [17, Lemma 3.3], but we describe the details here so that we can compare the construction with our functor $\mathcal{F}$ : Suppose $A=H^{-1}(E)$ for some $E \in \mathcal{E}_{0}$, and that $A$ is torsion-free with $A^{* *}$ locally free. For any surjection $q: \mathcal{E} x t^{1}\left(A, \mathcal{O}_{X}\right) \rightarrow Q$ in $\operatorname{Coh}(X)$ where $Q$ is 0-dimensional, let $K=\operatorname{ker}(q)$. We have a natural short exact sequence of sheaves

$$
0 \rightarrow A \stackrel{\beta}{\rightarrow} A^{* *} \stackrel{\gamma}{\rightarrow} T \rightarrow 0
$$

where $T \in \operatorname{Coh}^{\leq 1}(X)$. Taking derived dual and noting that $A^{* *}$ is locally free, we obtain the isomorphism

$$
\mathcal{E} x t^{1}\left(A, \mathcal{O}_{X}\right) \rightarrow \mathcal{E} x t^{2}\left(T, \mathcal{O}_{X}\right)
$$

where $\mathcal{E} x t^{2}\left(T, \mathcal{O}_{X}\right)$ is a pure sheaf in $\operatorname{Coh}^{\leq 1}(X)$ by [8, Proposition 1.1.6]. Dualising brings the short exact sequence

$$
0 \rightarrow K \rightarrow \mathcal{E} x t^{1}\left(A, \mathcal{O}_{X}\right) \stackrel{q}{\rightarrow} Q \rightarrow 0
$$

to the short exact sequence

$$
0 \rightarrow \mathcal{E} x t^{2}\left(\mathcal{E} x t^{1}\left(A, \mathcal{O}_{X}\right), \mathcal{O}_{X}\right) \stackrel{\delta}{\rightarrow} \mathcal{E} x t^{2}\left(K, \mathcal{O}_{X}\right) \stackrel{\epsilon}{\rightarrow} Q^{*} \rightarrow 0
$$


where we have an isomorphism $\mathcal{E} x t^{2}\left(\mathcal{E} x t^{2}\left(T, \mathcal{O}_{X}\right), \mathcal{O}_{X}\right) \stackrel{i}{\rightarrow} \mathcal{E} x t^{2}\left(\mathcal{E} x t^{1}\left(A, \mathcal{O}_{X}\right), \mathcal{O}_{X}\right)$. Note that if $T$ is nonzero, then it must be pure 1-dimensional. To see this, notice that the short exact sequence of sheaves 6.8.1) induces the short exact sequence in $\mathcal{A}^{p}$

$$
0 \rightarrow T \rightarrow A[1] \rightarrow A^{* *}[1] \rightarrow 0 .
$$

Since $\mathrm{Coh}^{\leq 0}(X)$ is a Serre subcategory of $\mathcal{A}^{p}$, any nonzero 0-dimensional subsheaf $T^{\prime}$ of $T$ would be an $\mathcal{A}^{p}$-subobject of $A[1]$, hence of $E$, contradicting the vanishing $\operatorname{Hom}\left(\operatorname{Coh}^{\leq 0}(X), E\right)=0$ from the definition of $\mathcal{E}_{0}$. Hence when $T$ is nonzero, it is pure 1-dimensional, hence reflexive [8, Proposition 1.1.10], and so the double dual map $T \stackrel{\alpha}{\rightarrow} \mathcal{E} x t^{2}\left(\mathcal{E} x t^{2}\left(T, \mathcal{O}_{X}\right), \mathcal{O}_{X}\right)=T^{* *}$ is an isomorphism. Putting everything together, the composition of morphisms in $\operatorname{Coh}(X)$

$$
A^{* *} \stackrel{\gamma}{\rightarrow} T \stackrel{\alpha}{\rightarrow} T^{* *}=\mathcal{E} x t^{2}\left(\mathcal{E} x t^{2}\left(T, \mathcal{O}_{X}\right), \mathcal{O}_{X}\right) \stackrel{i}{\rightarrow} \mathcal{E} x t^{2}\left(\mathcal{E} x t^{1}\left(A, \mathcal{O}_{X}\right), \mathcal{O}_{X}\right) \stackrel{\delta}{\rightarrow} \mathcal{E} x t^{2}\left(K, \mathcal{O}_{X}\right)
$$

has $A$ as the kernel and a 0-dimensional sheaf as the cokernel. Overall, we have constructed a 2-term complex

$$
A^{* *}=H^{-1}(E)^{* *} \stackrel{s}{\rightarrow} \mathcal{E} x t^{2}\left(K, \mathcal{O}_{X}\right)
$$

where $s=\delta i \alpha \gamma$. When $A=I_{C}$ is the ideal sheaf of a Cohen-Macaulay curve $C$ on $X$, we have $T=\mathcal{O}_{C}$, and the above construction reduces to the construction in 6.7 while the 2-term complex (6.8.2) coincides with the stable pair (6.7.2).

Recall that the proof of Lemma5.7describes a construction taking any element of $\mathcal{Q}\left(\mathcal{E} x t^{1}\left(I_{C}, \mathcal{O}_{X}\right)\right)$ to a rank-one PT stable object. The following lemma describes the precise relation between this construction in the proof of Lemma[5.7 and the construction in 6.8 .

Lemma 6.9. Let $E$ be an object of $\mathcal{E}_{0}$ such that $H^{-1}(E)$ is torsion-free and $H^{-1}(E)^{* *}$ is locally free. Suppose $q: \mathcal{E} x t^{1}\left(H^{-1}(E), \mathcal{O}_{X}\right) \rightarrow Q$ is a surjection in $\operatorname{Coh}(X)$ where $Q$ is 0 -dimensional. Let $G$ be the object in $\mathcal{E}_{0}$ satisfying $\mathcal{F}(G) \cong q$ as constructed in the proof of Lemma 5.7 and let $\left[H^{-1}(E)^{* *} \stackrel{s}{\rightarrow}\right.$ $\left.\mathcal{E}^{2} t^{2}\left(K, \mathcal{O}_{X}\right)\right]$ be the 2-term complex (6.8.2) constructed from $q$ as in 6.8. Then $G$ fits in an exact triangle

$$
\left(H^{-1}(E)^{*}\right)^{\vee} \stackrel{\phi}{\rightarrow} K^{\vee}[2] \rightarrow G \rightarrow\left(H^{-1}(E)^{*}\right)^{\vee}[1],
$$

and $s$ and $G$ are related by $H^{0}(\phi)=s$.

Proof. Let us use the notation in 6.8 and write $A=H^{-1}(E), K=\operatorname{ker}(q)$. Let us also write $c$ to denote the canonical map $A^{\vee}[1] \rightarrow \mathcal{E} x t^{1}\left(A, \mathcal{O}_{X}\right)$ as in the proof of Lemma[5.7, Let $C$ be an object that completes $q c$ to an exact triangle

$$
A^{\vee}[1] \stackrel{q c}{\rightarrow} Q \rightarrow C \rightarrow A^{\vee}[2] .
$$

Applying the octahedral axiom to the composition $q c$ then gives us the diagram

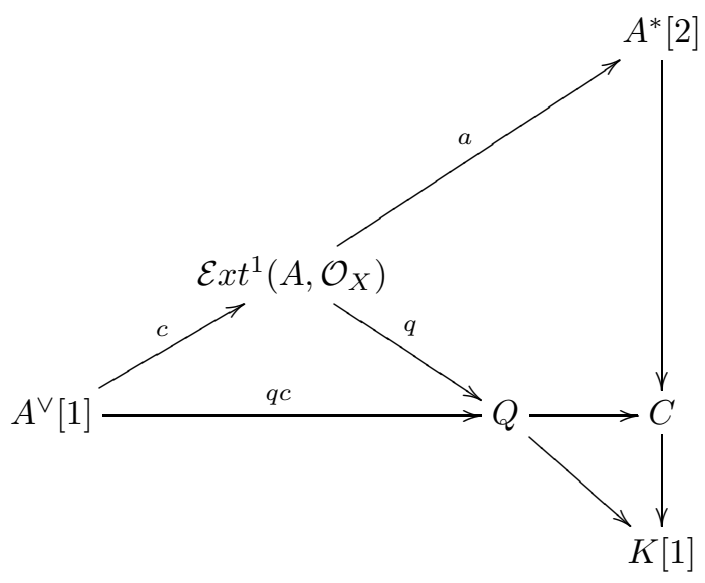


in which every straight line is an exact triangle. Taking $-{ }^{\vee}[3]$ then yields

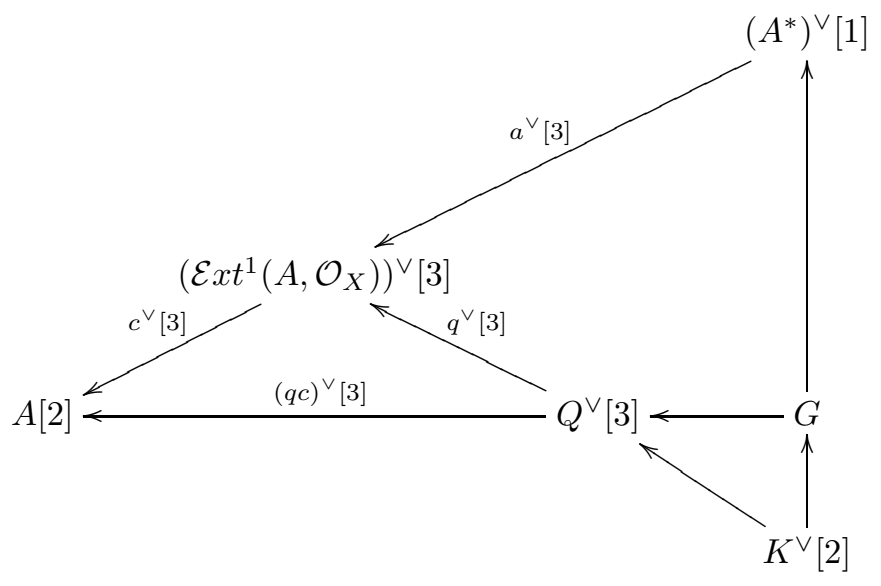

where we write $G=C^{\vee}[3]$. The vertical line in the last diagram gives an exact triangle

$$
\left(A^{*}\right)^{\vee} \stackrel{\phi}{\rightarrow} K^{\vee}[2] \rightarrow G \rightarrow\left(A^{*}\right)^{\vee}[1] .
$$

Now we apply the formulation of the octahedral axiom in [12, Lemma 1.4.6] to the composition $K^{\vee}[2] \rightarrow G \rightarrow Q^{\vee}[3]$, which yields the diagram

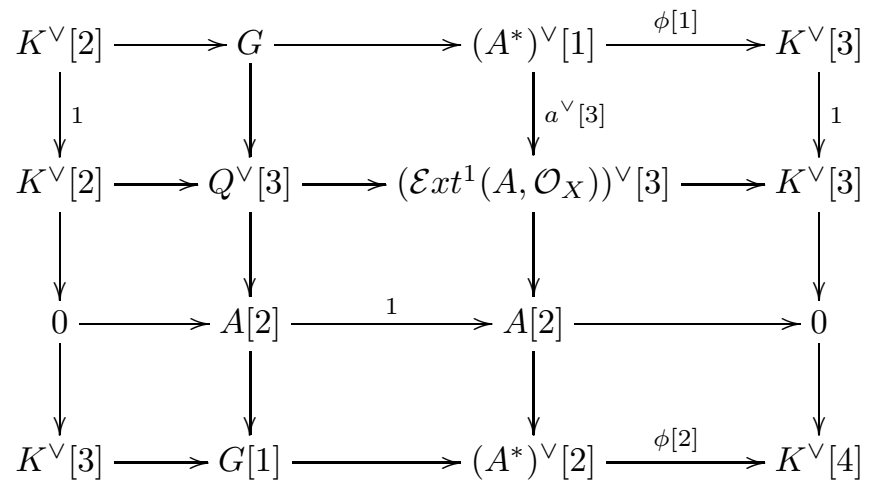

in which every row and every column is an exact triangle. Applying the cohomology functor $H^{-1}$ to the top-right square gives

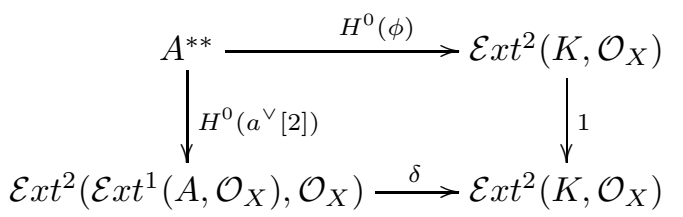

where $\delta$ is as in 6.8, and $H^{0}\left(a^{\vee}[2]\right)$ is precisely the surjection $i \alpha \gamma$ in 6.8. Thus $H^{0}(\phi)$ coincides with $s$, which was constructed as $\delta i \alpha \gamma$.

Example 6.10. Suppose $E$ is an object satisfying the hypotheses of Lemma 6.9, such that $H^{-1}(E)$ is not locally free while its dual $H^{-1}(E)^{*}$ is locally free. Using the notation in 6.8 and Lemma 6.9. we have that $T$ is nonzero and hence pure 1-dimensional by 6.8. It follows that $\mathcal{E}_{x} t^{1}\left(A, \mathcal{O}_{X}\right)$ and $K$ are both nonzero and pure 1-dimensional, as is $\mathcal{E} x t^{2}\left(K, \mathcal{O}_{X}\right)=K^{*}$. On the other hand, we have $\left(H^{-1}(E)^{*}\right)^{\vee}=H^{-1}(E)^{* *}$. Hence $\phi=H^{0}(\phi)$ and the morphism $\phi$ coincides with the morphism $s$ in Lemma 6.9. Exampes of such $E$ include: 
(1) $E$ is a rank-one PT stable object. In this case, we have $H^{-1}(E)=L \otimes I_{C}$ where $L$ is some line bundle and $I_{C}$ is the ideal sheaf of some Cohen-Macaulay curve $C$ on $X$, so that $\phi=s$ : $L \rightarrow \mathcal{E} x t^{2}\left(K, \mathcal{O}_{X}\right)$. When $L=\mathcal{O}_{X}$, this morphism is a PT stable pair.

(2) $E$ is a 2-term complex of coherent sheaves $[G \stackrel{\varphi}{\rightarrow} F]$ in $D^{b}(X)$ with $F$ sitting at degree 0 , and where $(G, F, \varphi)$ is a stable frozen triple (see Example 5.6). To see why $H^{-1}(E)$ satisfies the requirements of Lemma 6.9, consider the short exact sequence of sheaves

$$
0 \rightarrow H^{-1}(E) \rightarrow G \rightarrow \operatorname{im}(\varphi) \rightarrow 0
$$

where $\operatorname{im} \varphi$ is a pure 1-dimensional sheaf. This gives the isomorphism $\mathcal{E} x t^{1}\left(H^{-1}(E), \mathcal{O}_{X}\right) \cong$ $\mathcal{E} x t^{2}\left(\operatorname{im}(\varphi), \mathcal{O}_{X}\right) \neq 0$, and so $H^{-1}(E)$ cannot be locally free. We also have $H^{-1}(E)^{*}=$ $\mathcal{E} x t^{0}\left(H^{-1}(E), \mathcal{O}_{X}\right) \cong \mathcal{E} x t^{0}\left(G, \mathcal{O}_{X}\right)$, which is locally free. Hence $E$ satisfies all the requirements in Lemma 6.9

\section{REFERENCES}

[1] A. Bayer. Polynomial Bridgeland stability conditions and the large volume limit. Geom. Topol., 13:2389-2425, 2009.

[2] T. Bridgeland. Hall algebras and curve-counting invariants. J. Amer. Math. Soc., 24(4):969-998, 2011.

[3] W.-Y. Chuang and J. Lo. Stability and Fourier-Mukai transforms on higher dimensional elliptic fibrations. Comm. Anal. Geom., 24(5):1047-1084, 2016.

[4] S.I. Gelfand and Y.I. Manin. Methods of Homological Algebra. Springer Monographs in Mathematics. Springer Berlin Heidelberg, 2002.

[5] A. Gholampour and M. Kool. Higher rank sheaves on threefolds and functional equations. Preprint. arXiv:1706.05246 [math.AG], 2017.

[6] R. Hartshorne. Algebraic geometry. Springer-Verlag, New York, 1977. Graduate Texts in Mathematics, No. 52.

[7] D. Huybrechts. Fourier-Mukai Transforms in Algebraic Geometry. Oxford Mathematical Monographs. Clarendon Press, 2006.

[8] D. Huybrechts and M. Lehn. The Geometry of Moduli Spaces of Sheaves, volume 31 of Aspects of Mathematics. Vieweg, Braunschweig, 1997.

[9] J. Lo. Moduli of PT-semistable objects I. J. Algebra, 339(1):203-222, 2011.

[10] J. Lo. Polynomial bridgeland stable objects and reflexive sheaves. Math. Res. Lett., 19(4):873-885, 2012.

[11] J. Lo. Moduli of PT-semistable objects II. Trans. Amer. Math. Soc., 365(9):4539-4573, 2013.

[12] A. Neeman. Triangulated Categories. (AM-148). Annals of Mathematics Studies. Princeton University Press, 2014.

[13] R. Pandharipande and R. P. Thomas. Curve counting via stable pairs in the derived category. Invent. Math., 178:407-447, 2009.

[14] D. Piyaratne and Y. Toda. Moduli of Bridgeland semistable objects on 3-folds and Donaldson-Thomas invariants. J. Reine Angew. Math., 2015.

[15] A. Sheshmani. Higher rank stable pairs and virtual localization. Comm. in Anal. and Geom., 24(1):139-193, 2016.

[16] Y. Toda. Curve counting theories via stable objects I. DT/PT correspondence. J. Amer. Math. Soc., 23(4):1119-1157, 2010.

[17] Y. Toda. Hall algebras in the derived category and higher rank DT invariants. Preprint. arXiv:1601.07519 [math.AG], 2016.

Department of Mathematics, California State University, Northridge, 18111 Nordhoff Street, Northridge CA 91330, USA

E-mail address: jason.1o@csun.edu 